\title{
Acceleration of GRB outflows by Poynting flux dissipation
}

\author{
G. Drenkhahn* \\ Max-Planck-Institut für Astrophysik, Postfach 1317, 85741 Garching bei München, Germany
}

Received 21 December 2001 / Accepted 11 March 2002

\begin{abstract}
We study magnetically powered relativistic outflows in which a part of the magnetic energy is dissipated internally by reconnection. For GRB parameters, and assuming that the reconnection speed scales with the Alfvén speed, significant dissipation can take place both inside and outside the photosphere of the flow. The process leads to a steady increase of the flow Lorentz factor with radius. With an analytic model we show how the efficiency of this process depends on GRB parameters. Estimates are given for the thermal and non-thermal radiation expected to be emitted from the photosphere and the optically thin part of the flow respectively. A critical parameter of the model is the ratio of Poynting flux to kinetic energy flux at some initial radius of the flow. For a large value $(\gtrsim 100)$ the non-thermal radiation dominates over the thermal component. If the ratio is small ( $\lesssim 40)$ only prompt thermal emission is expected which can be identified with X-ray flashes.
\end{abstract}

Key words. gamma rays: bursts - magnetic fields - magnetohydrodynamics (MHD) - stars: winds, outflows

\section{Introduction}

To overcome the compactness problem of $\gamma$-ray bursts (GRBs) (e.g. Piran 1999) the central engines must produce radiating material moving ultra-relativistically fast towards the observer. GRB models must therefore describe an energy source which not only releases energy of around $10^{52} \mathrm{erg} / \mathrm{sterad}$ but must also explain the "clean" form of the energy. To produce the high Lorentz factors of the order of $10^{2}-10^{3}$ (Fenimore et al. 1993; Woods \& Loeb 1995; Lithwick \& Sari 2001) which are needed only a small fraction of the total energy can exist in form of rest mass energy of the matter involved.

The popular models involving compact objects or the collapse of a massive star to a black hole must include mechanisms how the energy is transported into a space region with few baryons. Otherwise large amounts of mass are expelled which cannot be accelerated to high Lorentz factors. The initially released energy could leave the central polluted region by neutrinos which annihilate to a pair plasma further away (Berezinskii \& Prilutskii 1987; Goodman et al. 1987; Ruffert et al. 1997). But due to the small cross section of neutrinos the efficiency is low and most of the energy escapes as neutrinos.

A Poynting flux dominating outflow will naturally occur if the compact object rotates and possesses a magnetic field. The luminosity will be fed by the rotational energy reservoir of the central object. Models involving an magnetised torus around a black hole (Mészáros \& Rees 1997) or a highly magnetised millisecond pulsar (Usov 1992;

\footnotetext{
* e-mail: georg@mpa-garching.mpg.de
}

Kluźniak \& Ruderman 1998; Spruit 1999) would produce such a rotationally driven Poynting flux. Extraction of energy from the central object by this magnetic process is potentially very efficient and fast.

In order to obtain not only a large energy extraction but also the observed large bulk Lorentz factors, the Poynting flux must be converted to kinetic energy. The simplest available magnetic acceleration models, in which the flow is approximated as radial, are problematic in this respect. In the classic non-relativistic case (Weber \& Davis 1967; Belcher \& MacGregor 1976) a dominating initial Poynting flux can transfer $1 / 3$ of its energy to the matter. If the flow is initially relativistic however almost no acceleration is possible (Michel 1969). The physical reason lies in the singular field and flow geometry of a purely radial flow. In this case the magnetic pressure gradient balances the magnetic tension force and no acceleration occurs. An imbalance between the pressure gradient and the tension force occurs in non-radial outflows, if the flow lines diverge faster with radius than in the radial case (Begelman \& Li 1994; Takahashi \& Shibata 1998). Detailed 1-dimensional calculations have been made which show how such a flow divergence can come about (Beskin 1997; Daigne \& Drenkhahn 2002).

In this paper we show that there is a second process which naturally leads to efficient conversion of Poynting flux to bulk kinetic energy. If the magnetic field in the outflow contains changes of direction on sufficiently small scales, (a part of) the magnetic energy is "free energy" which can be released locally in the flow by "fast reconnection" processes. Such a decay of magnetic energy, if it 
can occur rapidly enough, has two desirable effects. First it provides a source of energy outside the photosphere which is converted directly into radiation, without the relatively inefficient intermediate step of internal shocks (Spruit et al. 2001, hereafter Paper I). Secondly, it leads to an outward decrease of magnetic pressure, which causes a strong acceleration of the flow and conversion of Poynting flux to kinetic energy. In the present work, we concentrate on the acceleration effect, and show how it depends on the parameters (energy flux, baryon loading) of a GRB. This aspect of the model can be illustrated with analytic calculations. In a future paper we show, with more detailed numerical results, how the dissipated magnetic energy can also power the observed prompt radiation.

Changes of direction of field lines must occur in the flow in order for energy release by reconnection to be possible. These can occur naturally in a number of ways. If the magnetic field of a rotating central object is nonaxisymmetric the azimuthal part of the magnetic field in the flow changes direction on a length scale $\lambda \approx \pi v / \Omega$, where $v$ is the flow velocity and $\Omega$ the angular frequency. For an inclined dipole this yields the "striped" field in pulsar wind model of Coroniti (1990) where magnetic energy is released by the annihilation of the antiparallel field components. Field decay by reconnection was applied to pulsar winds (Coroniti 1990; Lyubarsky \& Kirk 2001) and also to GRBs (Thompson 1994; Paper I).

In this paper we investigate the dynamics of a magnetically powered outflow in which some of the energy dissipates by reconnection. With the assumption that the flow is highly dominated by magnetic energy and that the thermal energy is negligible we derive the velocity profile of the flow. The results provide estimates of the Lorentz factor of the flow, the photospheric radius, and the amount of energy that can be converted into non-thermal radiation. We investigate under which conditions prompt emission is expected and whether a considerable amount of thermal radiation can be produced. These predictions can then be tested against observations of the thermal component in GRB spectra (Preece 2000).

\section{Model description}

Highly magnetised spinning compact objects, e.g. millisecond pulsars or tori around black holes, are sources of Poynting flux that can power GRBs. They produce a plasma-loaded electromagnetic wind travelling outward and are fed by the rotational energy of the central object. In the wind of an aligned rotator the magnetic field is ordered and stationary. If ideal MHD applies, and the wind is radial in the poloidal plane, a large fraction of the total luminosity is bound to stay in form of Poynting flux. The picture changes in the case of an inclined rotator or any other source producing a non-axisymmetric rotating magnetic field. If the emitted Poynting flux contains modulations of the field it also carries along free magnetic energy, which can be extracted by reconnection processes. In these processes the field rearranges itself to an energetically preferred configuration while the energy released is transfered to the matter. Because perfect alignment of magnetic and rotation axis is a special case it is likely that most astrophysical objects produce modulated Poynting fluxes containing free magnetic energy.

A necessary condition for the existence of free magnetic energy in the flow is the field variation on small scales. For reconnection processes differently oriented field lines must come close to each other. Therefore the length scale on which the orientation of magnetic field lines change controls the speed of the field dissipation. The smaller the length scale is the faster the field can decay.

The general large scale magnetic field structure expected to be produced by a rotating object was discussed in Paper I. It is useful to consider simplified flow geometries along the equatorial plane and along the rotation axis as examples. In the equatorial plane an inclined rotator will produce a "striped" wind (Coroniti 1990). It consists of an electromagnetic wave in which the azimuthal field component varies with a wave length of $2 \pi c / \Omega$. Along the rotation axis the wave will have a circular component with the same wave length. Such wave-like field variations are present in general if a non-axisymmetric magnetic field component is present. The equatorial plane of an inclined rotator is only a prototype to illustrate the field geometry. In general, wave-like variations occur at all latitudes. If the rotator is aligned the field will be axisymmetric. Then, the magnetic field geometry looks like a wound up spiral on all cones of equal latitude. This field geometry is present in case of a jet-like outflow. Here, the magnetic field does not vary on small scales along the outflow direction. The differently directed field components lie on opposing sides of the rotation axis. In the context of a jet-like outflow the typical length scale of the field variation is the diameter of the jet cone $r \vartheta$ where $\vartheta$ is the jet opening angle.

In both of these field geometries MHD instabilities can promote reconnection processes. For wave-like variations current sheets form and tearing instability will lead to reconnection. For a polar jet-like outflow of an aligned rotator the field configuration is highly unstable to the kink instability (e.g. Bateman 1980, see also Paper I). It is plausible that the kink instability working in this case will distort the geometry after some time so that also wavelike variations come into play. This leads to non-periodic and highly irregular waves. Our model assumes the longitudinal field variation to be periodic so that the complicated effects of any non-periodicity is neglected. We consider both limiting cases for the small scale field variations though wave-like structures seem to be more general.

Near the source the flow is accelerated magnetocentrifugally (and perhaps thermally). It will be accelerated up to a distance around the Alfvén radius and then start to become radial asymptotically. The poloidal and azimuthal field components at the Alfvén radius are similar in magnitude. Beyond this point their ratio scales as $B_{\phi} / B_{\mathrm{r}} \sim r$, so that the radial component soon becomes negligible at a couple of Alfvén radii. The Alfvén point lies always inside the light radius $c / \Omega$ and if the magnetic field dominates, 
like in our case, the Alfvén radius and light radius are almost equal. Thus we can simplify the flow and field geometry at source distances $r>$ a few $\times c / \Omega \approx 10^{7} \mathrm{~cm}$ by assuming a purely radial flow with an azimuthal magnetic field. At this distance gravity effects can also be neglected. The magnetocentrifugal effects accelerate the flow to fast magneto-sonic velocity. Because we work in the cold limit (see below) and approximate the magnetic field to be purely poloidal the magneto-sonic velocity is equal to the Alfvén velocity. The initial flow velocity is set to the Alfvén velocity at some initial radius $r_{0} \gtrsim c / \Omega$.

To make a simple approach feasible analytically we have to make further approximations. The flow is treated stationary and its thermal energy is neglected ("cold" limit). This "cold" approximation is quite good in the optically thick region since no energy can be lost by radiation anyway. All of the dissipated energy is always converted into kinetic form. If the flow is optically thin the radiation produced by dissipation will freely escape and this energy part will not be converted into kinetic energy. Our model overestimates the kinetic energy gained in the optically thin regime. Statements about the radius of the photosphere, where the flow changes from optically thick to thin, or the Lorentz factor there will hold rather robustly.

\subsection{Magnetic field dissipation}

The dissipation is modelled by using the typical length scale of the magnetic field $\lambda$ and a fraction of the Alfvénic velocity $\epsilon v_{\mathrm{A}}$ where $\epsilon$ is an dimensionless factor. The idea is that the magnetic field lines with different directions get advected towards a reconnection centre where the field dissipates (Petschek 1964). This advection happens with a fraction of $v_{\mathrm{A}}$. The decay of mean field must also depend on $v_{\mathrm{A}}$ in a similar fashion. Though there are elaborate models on the reconnection physics (e.g. Coroniti 1990; Thompson 1994) we prefer to express this rather uncertain topic in form of the dimensionless free parameter $\epsilon$ and the Alfvénic speed $v_{\mathrm{A}}$. All the uncertain physics in this picture is taken up by $\epsilon$. Our ansatz for the time scale of the mean field decay, in a comoving frame is then

$\tau_{\mathrm{co}}=\frac{\lambda_{\mathrm{co}}}{\epsilon v_{\mathrm{A}, \mathrm{co}}}$

where quantities considered in the comoving frame are indexed with "co". This comoving frame moves with the mean large scale bulk flow motion so that the small scale motion is neglected.

The reconnection takes place at certain reconnection centres in the flow. The typical distance between these reconnection centres also influence the rate of the overall field dissipation. Since we regard the field dissipation in all generality and for a variety of field geometries we cannot explicitely model these small scale details about the density of reconnection centres. This uncertain issue must also be handled by the free parameter $\epsilon$ so that $\epsilon$ controls the average field dissipation on larger length scales.
At first sight $\epsilon<1$ is an upper limit since for $\epsilon=1$ reconnection would happen everywhere with an advection speed of $c$. If the advection towards the reconnection centres happens with almost $c$ large current densities are required. The MHD condition might break down leading to an additional decay of magnetic field. This effect could be parameterised by an larger value of $\epsilon$ so that an upper limit of 1 may not be strict.

For most of the paper we will work with a fiducial value of $\epsilon=0.1$. One should keep in mind that $\epsilon$ is perhaps the most uncertain quantity of the model because it may not be constant and its value cannot be estimated by first principles in general.

The length scale for the dissipation $\lambda_{\text {co }}$ depends on the nature of the outflow as discussed in the last section. We will distinguish the two cases where the field variation is encountered on length scales longitudinal to the flow direction (called longitudinal case in the rest of the paper) and where this length scale is transversal to the flow direction (transversal case). The transversal case is found in a polar outflow of a aligned rotator where the field components having different directions lie on opposite sides of the rotation axis. For mathematical simplicity we will regard here the two limiting cases only and make a few notes on the mixed case later in this study.

The longitudinal and the transversal length scales $\lambda_{\mathrm{lo}}, \lambda_{\mathrm{tr}}$ in the comoving frame scale differently with the Lorentz factor of the flow $\Gamma$ :

$\lambda_{\mathrm{lo}, \mathrm{co}}=\frac{2 \pi c \Gamma}{\Omega}$

$\lambda_{\mathrm{tr}, \mathrm{co}}=\vartheta r$

where $\vartheta$ is some kind of an opening angle of the polar outflow. $\lambda_{\text {tr.co }}$ does not scales with $\Gamma$ because it denotes a length which is perpendicular to the direction of motion being the same in the lab and comoving frame.

\section{The dynamics of the flow}

The reconnection processes described in the last section will change constantly the structure of the magnetic field on a length scale of the order of the wave length (small length scale of the problem). Though, the azimuthal field line stretching will keep the field aligned predominantly perpendicular to the flow direction. The exact field structure is not important because only the magnetic energy density $B^{2} /(8 \pi)$ enters the dynamic equations. In the following, $\boldsymbol{B}$ denotes the dynamical effective transversal magnetic field which is constant over small scales. The induction equation will still be valid for the effective field.

\subsection{Conservation laws}

The dynamics of the flow is governed by the ideal MHD equations for the conservation of energy, momentum and mass. For the relativistic treatment the equations are 
formally best written in tensorial form (e.g. Bekenstein \& Oron 1978):

$T_{; \nu}^{\mu \nu}=0$,

$\left(\rho u^{\mu}\right)_{; \mu}=0$

where

$$
\begin{aligned}
T^{\mu \nu}= & \underbrace{w u^{\mu} u^{\nu}+p g^{\mu \nu}}_{\text {matter part }} \\
& +\underbrace{\frac{1}{4 \pi}\left[\left(u^{\mu} u^{\nu}+\frac{1}{2} g^{\mu \nu}\right) b_{\alpha} b^{\alpha}-b^{\mu} b^{\nu}\right]}_{\text {electromagnetic part }}
\end{aligned}
$$

is the energy-momentum tensor for ideal MHD. The signature $(-+++)$ is used for the metric tensor $g^{\mu \nu}$. Here, $\rho, w$ and $p$ are the mass density, the enthalpy density and the pressure in the proper frame of the fluid moving with a 4 -velocity $u^{\mu}=(\Gamma, \boldsymbol{u}) \cdot b^{\mu}={ }^{*} F^{\mu \nu} u_{\nu}$ is the the 4 -vector of the magnetic field where ${ }^{*} F^{\mu \nu}$ is the dual electromagnetic field strength tensor.

Now we choose a spherical coordinate system centred on the central engine. The flow is assumed to be spherically symmetric and the field dominated by its toroidal component. In this case $\boldsymbol{u} \perp \boldsymbol{B}$ and the components of the magnetic four vector are simply $b^{\mu}=(0,-\boldsymbol{B} / \Gamma)$ and $b_{\mu} b^{\mu}=B_{\mathrm{co}}^{2}=B^{2} / \Gamma^{2}$. Writing (4) and (5) in coordinate form and assuming stationarity gives the conservation laws for energy, momentum and mass

$0=\partial_{r} r^{2}\left(w \Gamma u+\frac{\beta B^{2}}{4 \pi}\right)$,

$0=\partial_{r} r^{2}\left(w u^{2}+\left(1+\beta^{2}\right) \frac{B^{2}}{8 \pi}\right)+r^{2} \partial_{r} p$

$0=\partial_{r} r^{2} \rho u$

where $\beta=u / \Gamma$ (Königl \& Granot 2002; Lyutikov 2001). By integrating the mass and energy equations one obtains the total mass loss per time per sterad

$\dot{M}=r^{2} \rho u c$

and the total luminosity per sterad

$L=r^{2}\left(w \Gamma u c+\frac{\beta B^{2}}{4 \pi} c\right)$.

The enthalpy density $w$ includes the rest mass energy density $\rho c^{2}$. In the following we will assume a cold flow with $w=\rho c^{2}, p=0$. Then, the momentum equation can be integrated and the conservation laws read

$$
\begin{aligned}
& \dot{M}=r^{2} \rho u c, \\
& L=\Gamma \dot{M} c^{2}+\beta c \frac{(r B)^{2}}{4 \pi}, \\
& \text { const. }=u \dot{M} c+\left(1+\beta^{2}\right) \frac{(r B)^{2}}{8 \pi} .
\end{aligned}
$$

In the energy Eq. (13) one can identify the kinetic energy flux per sterad $L_{\text {kin }}=\Gamma \dot{M} c^{2}$ and the Poynting luminosity per sterad $L_{\mathrm{pf}}=\beta c(r B)^{2} /(4 \pi)$.
Taking the flow to be cold and eliminating $(r B)^{2}$ from (13), (14) shows that $u$ is a constant function of $r$. Finding an exact accelerating solution of the energy and momentum equations without thermal pressure is not possible. By using an evolution equation for the magnetic field $B$ (see Sect. 3.4 below) and combining it with the energy Eq. (13) one obtains an accelerating solution but violates the momentum conservation. Luckily, the error made by that becomes small in the ultra-relativistic limit. Then, $\Gamma \approx u, \beta \approx 1$ so that the first term in the momentum Eq. (8) becomes small since it approaches the form of the rhs of the energy Eq. (7). As a consequence the thermal pressure gradient term of (8) must also be small. Setting the pressure to zero and solving the energy equation means that the momentum equation is almost satisfied. Since we only consider ultra-relativistic flows the error made is small which justifies the use of the cold approximation.

\subsection{A note on the ideal MHD approximation}

In the treatment above the ideal MHD approximation was used. But a key ingredient of the model is the existence of field dissipation for which ideal MHD is not applicable at first sight. The field dissipation acts like an effective diffusivity in the plasma so that the effective mean electric field in a comoving frame does not vanish. Since a substantial electric field $\boldsymbol{E}_{\text {co }}$ would contribute to the comoving energy density, the question arises if it can be neglected. We found from a more detailed numerical investigation (in preparation) that the comoving electric field is in fact small, and we use this advance knowledge to neglect its contribution to the dynamics here.

\subsection{The initial conditions of the flow}

Let $\sigma$ be the ratio of Poynting flux to matter energy flux:

$\sigma=\frac{L_{\mathrm{pf}}}{L_{\mathrm{kin}}}=\frac{\beta(r B)^{2}}{4 \pi \Gamma \dot{M} c}=\frac{B^{2}}{4 \pi \Gamma^{2} \rho c^{2}}$.

$\sigma$ is also the magnetisation parameter of the plasma, describing the ratio of the proper magnetic energy density to the proper energy density of the matter. The Alfvén 4 -velocity in the comoving frame is

$u_{\mathrm{A}}=\frac{B / \Gamma}{\sqrt{4 \pi \rho c^{2}}}=\sqrt{\sigma}$

with the regular dimensional velocity counterpart

$v_{\mathrm{A}}=c \frac{u_{\mathrm{A}}}{\sqrt{1+u_{\mathrm{A}}^{2}}}=c \sqrt{\frac{\sigma}{1+\sigma}}$.

At an initial radius $r_{0}$, where the flow starts with the Alfvén speed (discussed in Sect. 2), the relation between initial 4 -velocity $u_{0}$ and initial Poynting flux ratio $\sigma_{0}$ is simply

$u_{0}=u\left(r_{0}\right)=u_{\mathrm{A}}\left(r_{0}\right)=\sqrt{\sigma_{0}}$. 
The total energy and the mass flux are linked by

$L=\left(\sigma_{0}+1\right) \Gamma_{0} \dot{M} c^{2}$

so that $\dot{M}$ can be expressed in terms of $L$ and $\sigma_{0}$ :

$\dot{M}=\frac{L}{c^{2}\left(\sigma_{0}+1\right)^{3 / 2}}$.

In the GRB case the flow must start highly Poynting flux dominated with $\sigma_{0} \gtrsim 100$ so that $u \gg 1$ at all distances. One can therefore set $\sigma_{0}+1 \approx \sigma_{0}, u \approx \Gamma$ and $\beta \approx 1$. The conservation Eqs. (12)-(14) then reduce to

$\dot{M}=r^{2} \rho u c$,

$L\left(1-\frac{u}{\sigma_{0}^{3 / 2}}\right)=c \frac{(r B)^{2}}{4 \pi}$.

In this limit one can also simplify the expression (16) for the Alfvén speed in the comoving frame by using (21) and (21):

$\beta_{\mathrm{A}}=\sqrt{1-\frac{u}{\sigma_{0}^{3 / 2}}}, \quad u_{\mathrm{A}}=\frac{\sigma_{0}^{3 / 2}}{u}$.

Equation (21) states that there is no acceleration if $B \sim$ $1 / r$. This is the case in a radial outflow with ideal MHD conditions. We encounter here again the fact that the Poynting flux energy in a radial ultra-relativistic MHD outflow cannot be transfered to the matter (e.g. Begelman \& Li 1994; Daigne \& Drenkhahn 2002).

\subsection{The evolution of the magnetic field}

The evolution of the magnetic field, as it is carried with the flow, is governed by the induction equation. This includes the effects of advection and field line stretching. In addition we will include a term to describe the decay of (a part of) the field by reconnection as described in Sect. 2.1. Since the reconnection is easiest described in a local, comoving frame, we first transform the induction equation

$\partial_{t} \boldsymbol{B}=-c \operatorname{curl} \boldsymbol{E}$

into the comoving frame where we extend it to account for the reconnection.

In the stationary case of our model setup the induction equation for ideal MHD is

$\partial_{r} \beta r B=0$.

This equation describes the field evolution due to ideal MHD processes. To obtain the evolution term in the the comoving frame we first need the convective derivative

$\frac{\mathrm{d} B}{\mathrm{~d} t}=c \beta \partial_{r} B=-\frac{c B}{r} \partial_{r} r \beta$ which then gives in terms of the comoving quantities

$$
\begin{aligned}
\frac{\mathrm{d} B}{\mathrm{~d} t} & =\frac{\mathrm{d}\left(\Gamma B_{\mathrm{co}}\right)}{\Gamma \mathrm{d} t_{\mathrm{co}}} \\
& =\frac{\mathrm{d} B_{\mathrm{co}}}{\mathrm{d} t_{\mathrm{co}}}+\frac{B_{\mathrm{co}}}{\Gamma} \frac{\mathrm{d} \Gamma}{\mathrm{d} t_{\mathrm{co}}} \\
& =\frac{\mathrm{d} B_{\mathrm{co}}}{\mathrm{d} t_{\mathrm{co}}}+\frac{B}{\Gamma} \frac{\mathrm{d} \Gamma}{\mathrm{d} t}
\end{aligned}
$$

Combining (26) and (29) gives the comoving field evolution without dissipation effects

$\frac{\mathrm{d} B_{\mathrm{co}}}{\mathrm{d} t_{\mathrm{co}}}=-\frac{c B}{\Gamma r} \partial_{r} r u$.

Let us denote the striped, decayable part of the magnetic field with $\boldsymbol{B}_{\rightleftharpoons}$ and the perpendicular, non-reconnecting part with $\boldsymbol{B}_{\Uparrow}$. We model the decay of the striped component of the magnetic field in the comoving frame by the ansatz

$\frac{\mathrm{d} B_{\rightleftharpoons \mathrm{co}}}{\mathrm{d} t_{\mathrm{co}}}=-\frac{c B_{\rightleftharpoons}}{\Gamma r} \partial_{r} r u-\frac{B_{\rightleftharpoons}}{\tau}$

where $\tau$ is the field decay time scale from (1) in the lab frame. The non-decaying part $\boldsymbol{B}_{\Uparrow}$ evolves according to the induction Eq. (25) so that

$\beta r B_{\Uparrow}=$ const.

Expressing the comoving quantities in terms of lab frame quantities similar to (27)-(29) gives

$\frac{\mathrm{d} B \rightleftharpoons \mathrm{co}}{\mathrm{d} t_{\mathrm{co}}}=\frac{\mathrm{d} B_{\rightleftharpoons}}{\mathrm{d} t}-\frac{B_{\rightleftharpoons}}{\Gamma} \frac{\mathrm{d} \Gamma}{\mathrm{d} t}$.

Since the flow is stationary we can replace the convective derivatives by $r$-derivatives and after combining (31) and (33) one obtains

$c \beta \partial_{r} B \rightleftharpoons-\frac{B_{\rightleftharpoons}}{\Gamma} c \beta \partial_{r} \Gamma=-\frac{c B_{\rightleftharpoons}}{\Gamma r} \partial_{r} r u-\frac{B_{\rightleftharpoons}}{\tau}$.

One arrives at

$\partial_{r} \beta r B_{\rightleftharpoons}=\frac{r B_{\rightleftharpoons}}{c \tau}$

and with $\beta r B_{\rightleftharpoons}=\sqrt{(\beta r B)^{2}-\left(\beta r B_{\Uparrow}\right)^{2}}$ this yields

$\partial_{r} \beta r B=-\frac{r B}{c \tau}\left[1-\left(\frac{B_{\Uparrow}}{B}\right)^{2}\right]$.

Let $\mu=\left(B_{\Uparrow} / B\right)_{0}$ so that $\mu$ is the initial fraction between the field strengths of the non-decaying component and the total field. $\mu=0$ means that the complete field decays while at $\mu=1$ only ideal MHD processes occur and no dissipation takes place. In the case of an equatorial outflow of an inclined rotator $\mu=\cos i$ where $i$ is the inclination. The other cases are more complicated and $\mu$ is not associated to a simple geometric quantity.

Using $\mu$ as a constant of the problem the field decay equation can be cast into this simple form:

$\partial_{r}(\beta r B)^{2}=-2 \frac{(\beta r B)^{2}}{\beta c \tau}\left[1-\mu^{2} \frac{(\beta r B)_{0}^{2}}{(\beta r B)^{2}}\right]$ 
Equations (12), (13), (14), (37) over-determine the 3 unknown functions $\rho, u, B$ because we have assumed a cold flow so that the internal energy is neglected. But in the ultra relativistic limit the energy and momentum Eqs. (13), (14) are equal. In this limit (21), (21), (37) are sufficient to obtain the solutions for $\rho, u, B$.

\subsection{Solution of the flow problem}

In the ultra relativistic limit $\beta \approx 1, \Gamma \approx u$ Eq. (37) for the evolution of the magnetic field with distance, including dissipation becomes

$\partial_{r}(r B)^{2}=-2 \frac{(r B)^{2}}{c \tau}\left[1-\mu^{2} \frac{(r B)_{0}^{2}}{(r B)^{2}}\right]$.

Using the $B-u$ relation (21) to eliminate $(r B)^{2}$ one obtains a differential equation for $u$ :

$\partial_{r} u=\frac{2}{c \tau}\left[\sigma_{0}^{3 / 2}\left(1-\mu^{2}\right)+\sqrt{\sigma_{0}} \mu^{2}-u\right]$.

This equation is to be integrated with initial condition $u=u_{0}=\sqrt{\sigma}$. In the absence of internal dissipation $(\mu=$ 1 ) the flow is not accelerated, $\partial_{r} u=0$, as expected. The flow accelerates monotonically and reaches asymptotically its terminal speed $u_{\infty}$ found be setting $\partial_{r} u=0$ :

$u_{\infty}=\sigma_{0}^{3 / 2}\left(1-\mu^{2}\right)+\sqrt{\sigma_{0}} \mu^{2}$.

The dissipation time scales are

$\tau_{\mathrm{lo}}=\frac{2 \pi}{\epsilon \Omega} \frac{u^{2}}{\sqrt{1-u / \sigma_{0}^{3 / 2}}}$

and

$\tau_{\mathrm{tr}}=\frac{\vartheta}{\epsilon c} \frac{r u}{\sqrt{1-u / \sigma_{0}^{3 / 2}}}$

for the longitudinal and transversal cases from (1), (2), (3), (23).

Since our model rests on the assumption of a significant "decayable" component, $\mu$ in the following is taken to be of the order 0.5 but not close to 1 . Then, the terminal velocity $u_{\infty}$ is much larger than the initial velocity and $u_{\infty} / u_{0} \approx \sigma_{0}\left(1-\mu^{2}\right) \gg 1$ and (40) simplifies to

$u_{\infty} \approx\left(1-\mu^{2}\right) \sigma_{0}^{3 / 2}$.

The differential Eq. (39) is analytically solvable at intermediate source distances, where the flow is much faster than the initial velocity $\left(\sqrt{\sigma_{0}} \ll u\right)$ but is still far away from the point where the acceleration saturates $(u \ll$ $u_{\infty}<\sigma_{0}^{3 / 2}$ ). The dissipation time scales then simplify to

$\tau_{\mathrm{lo}} \approx \frac{2 \pi u^{2}}{\epsilon \Omega}, \quad \tau_{\mathrm{tr}} \approx \frac{\vartheta r u}{\epsilon c}$.

In this case $u_{\infty}-u \approx u_{\infty}$ and (39) becomes

$\partial_{r} u=\frac{2 u_{\infty}}{c \tau}$

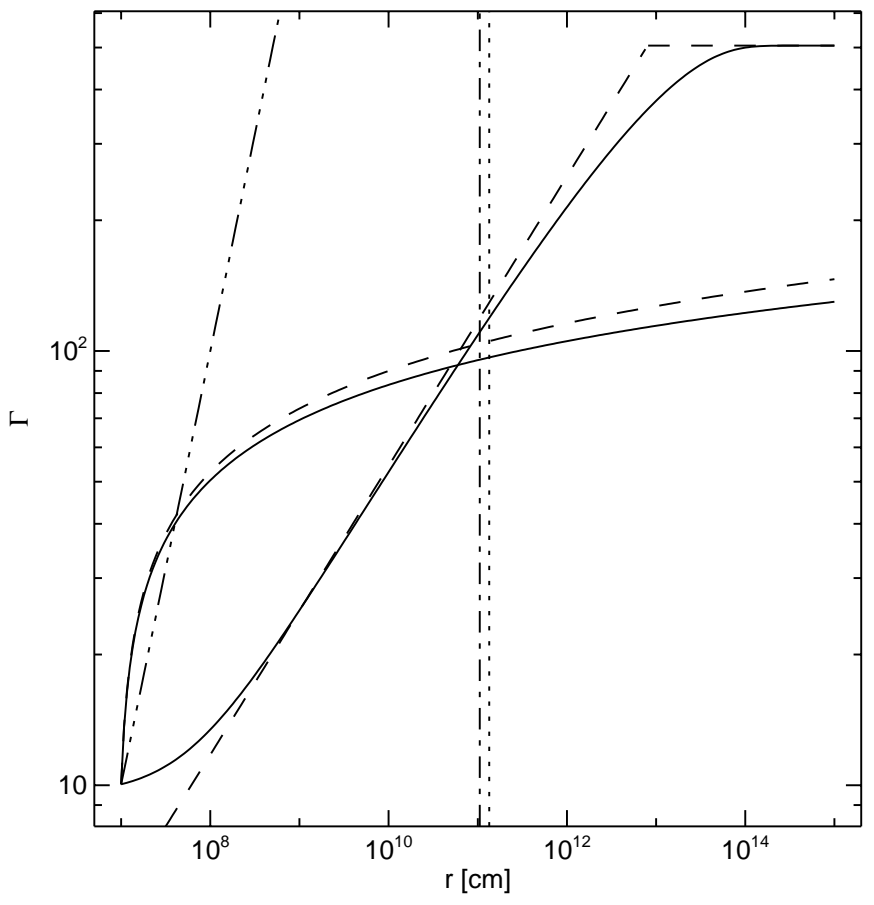

Fig. 1. Lorentz factor $\Gamma$ of the flow as function of radius $r$ for the longitudinal and transversal cases. Model parameters are $\sigma_{0}=100, \epsilon=0.1, \mu^{2}=0.5, \Omega=10^{4} \mathrm{~s}^{-1}, \vartheta=10^{\circ}$ and $r_{0}=10^{7} \mathrm{~cm}$. The solid lines are the numerical solutions of (39) while the dashed lines are the approximations (47) and (48). The vertical lines correspond to the photospheric radii: dotted for the transversal case and dashed-dotted for the longitudinal case model. The steep dashed-dotted line represents the $u \sim r$ law which is expected in the classic non-magnetic optically thick fireball models (Paczyński 1986).

with the solutions

$u=\left(\frac{3}{\pi c} \epsilon \Omega u_{\infty}\left(r-r_{0}\right)+\sigma_{0}^{3 / 2}\right)^{1 / 3} \quad$ (long. case)

and

$u=\left(\frac{4 \epsilon u_{\infty}}{\vartheta} \ln \left(\frac{r}{r_{0}}\right)+\sigma_{0}\right)^{1 / 2} \quad$ (transversal case).

The function $u$ for the longitudinal case can be described as a broken power-law as can be seen in Fig. 1. In the domain $r_{0} \ll r, u_{0} \ll u \ll u_{\infty}$, which we have regarded anyway for finding the solution, the 4 -velocity is well approximated by

$u=\left(\frac{3}{\pi c} \epsilon \Omega u_{\infty} r\right)^{1 / 3}$.

\subsection{The length scale for the acceleration}

In the longitudinal case the dissipation stops approximately where the rising power-law part of the 
$u$ functions (48) reaches the $u_{\infty}$ limit (43). By using (43) and (48) one can write down this saturation radius as

$$
\begin{aligned}
r_{\mathrm{sr}} & =\frac{\pi c}{3} \frac{u_{\infty}^{2}}{\epsilon \Omega}=\frac{\pi c}{3} \frac{\sigma_{0}^{3}\left(1-\mu^{2}\right)^{2}}{\epsilon \Omega} \\
& =7.85 \times 10^{12} \mathrm{~cm} \cdot\left(\epsilon_{-1} \Omega_{4}\right)^{-1} \sigma_{0,2}^{3}\left(\frac{1-\mu^{2}}{0.5}\right)^{2} .
\end{aligned}
$$

The complete analytical approximation for the longitudinal case reads

$u=\left\{\begin{array}{ll}u_{\infty}\left(r / r_{\mathrm{sr}}\right)^{1 / 3} & \text { for } r \leq r_{\mathrm{sr}} \\ u_{\infty} & \text { for } r>r_{\mathrm{sr}}\end{array}\right.$.

\section{Analysis of the results}

\subsection{The photospheric radius}

The photosphere is located where the optical depth reaches a value of 1 . The optical depth depends on the density and the the radial velocity $u$ and must be integrated from a finite radius to infinity. Because we only want to know the photospheric radius within a factor of, say 2 , we define it to be where the mean free path of a photon equals the distance from the source $r$. In the comoving frame a photon sees the mass density $\rho$ and the mean free path for Thompson scattering is $1 /(\kappa \rho)$. The source distance in this frame is $r / \Gamma$ so that the photosphere is located at

$r_{\mathrm{ph}}=\frac{u_{\mathrm{ph}}}{\kappa \rho_{\mathrm{ph}}}$

which yields

$$
\frac{\kappa L}{c^{3} \sigma_{0}^{3 / 2}}=u_{\mathrm{ph}}^{2} r_{\mathrm{ph}} \text {. }
$$

If we neglect not only the initial velocity but also the initial radius $r_{0}$ compared to the photospheric radius, (46) and (47) simplify to

$u_{\mathrm{ph}}^{3}=\frac{3}{\pi c} \epsilon \Omega\left(1-\mu^{2}\right) \sigma_{0}^{3 / 2} r_{\mathrm{ph}} \quad$ (longitudinal case),

$u_{\mathrm{ph}}^{2}=\frac{4 \epsilon\left(1-\mu^{2}\right) \sigma_{0}^{3 / 2}}{\vartheta} \ln \left(\frac{r_{\mathrm{ph}}}{r_{0}}\right) \quad$ (transversal case).

Together with condition (52) at the photosphere we arrive at the equations for the photospheric radius and the 4velocity in the longitudinal case

$$
\begin{aligned}
u_{\mathrm{ph}}= & {\left[\frac{3 \kappa}{\pi c^{4}} \epsilon \Omega\left(1-\mu^{2}\right) L\right]^{1 / 5} } \\
= & 119 \cdot\left[\epsilon_{-1} \Omega_{4}\left(\frac{1-\mu^{2}}{0.5}\right) L_{50}\right]^{1 / 5}, \\
r_{\mathrm{ph}}= & {\left[\frac{\pi^{2} \kappa^{3}}{9 c^{7}} \frac{L^{3}}{\left(\epsilon \Omega\left(1-\mu^{2}\right)\right)^{2}}\right]^{1 / 5} \sigma_{0}^{-3 / 2} } \\
= & 1.05 \times 10^{11} \mathrm{~cm} \\
& \times\left[\epsilon_{-1} \Omega_{4}\left(\frac{1-\mu^{2}}{0.5}\right)\right]^{-2 / 5} L_{50}^{3 / 5} \sigma_{0,2}^{-3 / 2} .
\end{aligned}
$$

Note that the 4 -velocity at the photosphere $u_{\mathrm{ph}}$ does not depend on the initial Poynting flux ratio $\sigma_{0}$ and only weakly on $L$.

For the transversal case the flow velocity always depends greatly on the initial radius $r_{0}$. The dissipation time scale is $\tau_{\text {tr }} \sim r u$ and most energy is released at small $r$ near the source. The acceleration depends crucially on the onset of the dissipation and therefore on $r_{0}$. In our simple model $r_{0}$ and $\vartheta$ are not well determined by physical arguments so that the transversal case is rather uncertain and highly speculative. One cannot write down robust equations for the photosphere like in the longitudinal case without many degrees of freedom.

\subsection{Energy available for prompt radiation}

The energy dissipated beyond the photospheric radius is

$L_{\mathrm{D}}=\left(u_{\infty}-u_{\mathrm{ph}}\right) \dot{M} c^{2}$.

Using (20), (43) and (55) this yields

$L_{\mathrm{D}}=e\left(1-\mu^{2}\right) L$

with

$e=1-\left(\frac{3 \kappa}{\pi c^{4}} \frac{\epsilon \Omega L}{\left(1-\mu^{2}\right)^{4}}\right)^{1 / 5} \sigma_{0}^{-3 / 2}$

For a Poynting flux dominated flow, the magnetic energy flux equals the total energy flux $L$. Of this, a fraction $1-\mu^{2}$ is dissipated internally while a fraction $e\left(1-\mu^{2}\right)$ can be converted to radiation beyond the photosphere. Thus $e$ is an efficiency factor, which gives the ratio between the energy dissipated in the optically thin domain and the total dissipated energy. Efficient conversion of free magnetic energy into non-thermal radiation can happen if $e$ is of the order unity which requires that the second term in (59) is small:

$0.24 \times\left(\epsilon_{-1} \Omega_{4} L_{50}\right)^{1 / 5}\left(\frac{1-\mu^{2}}{0.5}\right)^{-4 / 5} \sigma_{0,2}^{-3 / 2}<1$

or written differently

$\sigma_{0}>39 \times\left(\epsilon_{-1} \Omega_{4} L_{50}\right)^{2 / 15}\left(\frac{1-\mu^{2}}{0.5}\right)^{-8 / 15}$

where $\epsilon_{-1}=\epsilon / 0.1, \quad \Omega_{4}=\Omega /\left(10^{4} \mathrm{~s}^{-1}\right), \quad L_{50}=$ $L /\left(10^{50} \mathrm{erg} \mathrm{s}^{-1}\right.$ sterad $\left.^{-1}\right)$ and $\sigma_{0,2}=\sigma_{0} / 100$ are parameters scaled to fiducial GRB values.

When (61) is satisfied, part of the magnetic energy is released beyond the photosphere, and powers the prompt radiation. If it is not satisfied, the energy is released inside the photosphere and is converted, instead, into bulk kinetic energy. Some other means of conversation into radiation is then needed, such as internal shocks. Since the dependence on parameters other than the initial Poynting flux ratio $\sigma_{0}$ is small in (61), we conclude that efficient powering of prompt radiation by magnetic dissipation in GRB is possible for $\sigma_{0} \gtrsim 100$. 


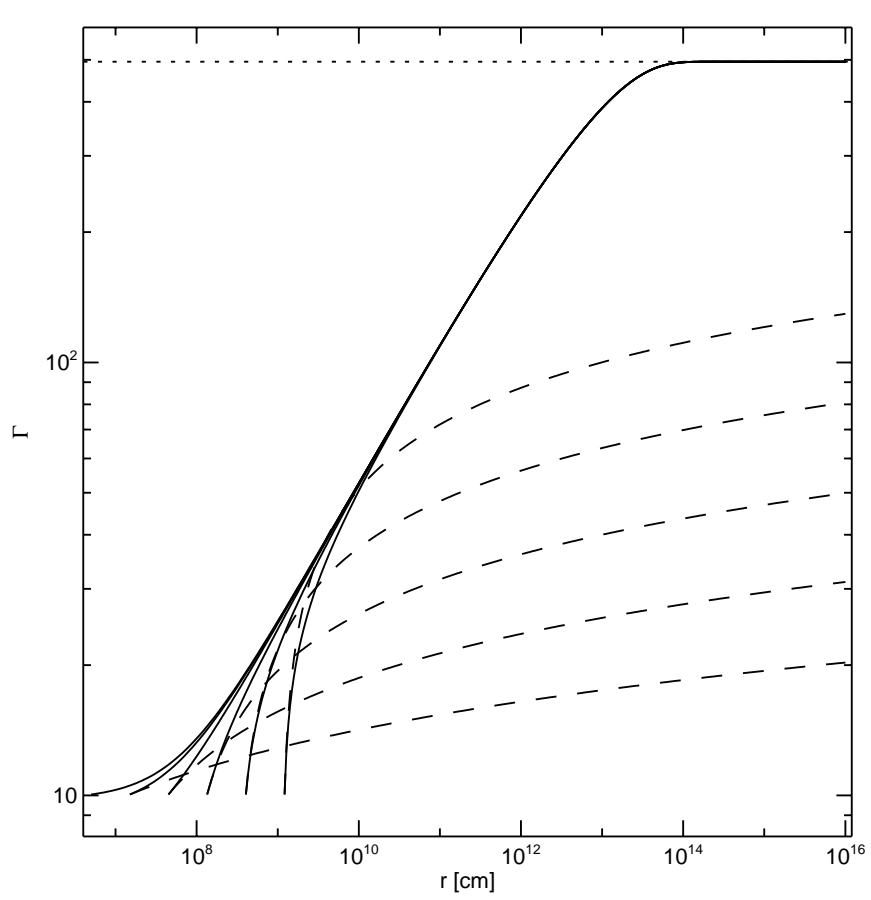

Fig. 2. The influence of $r_{0}$ on the Lorentz factor: the solid lines correspond to longitudinal case solutions and the dashed one to transversal case solutions with $1.5 \times 10^{7} \mathrm{~cm} \leq r_{0} \leq 1.2 \times 10^{9} \mathrm{~cm}$. The constants used where $\sigma_{0}=100, \mu^{2}=0.5, \epsilon=0.1, \Omega=$ $10^{4} \mathrm{~s}^{-1}$. For the transversal cases $\vartheta=2 \pi c \sqrt{\sigma_{0}} /\left(\Omega r_{0}\right)$ is chosen so that the initial acceleration (slope at $r_{0}$ ) is the same as in the corresponding longitudinal cases.

\subsection{Longitudinal and transversal cases in comparison}

The major difference between the longitudinal and the transversal case is the different dissipation time scale. While the decay time scale for the longitudinal case (44) is $\tau_{\text {lo }} \sim u^{2}$ and therefore limited by $u \leq u_{\infty}$, the time scale for the transversal case $\tau_{\operatorname{tr}} \sim r u$ is not limited. At small radii it starts at low values but grows then to infinity. This major difference is visualised in Fig. 1 where the flow Lorentz factor is plotted depending on the radius.

As mentioned in Sect. 4.1 the transversal case depends strongly on the initial radius $r_{0}$. This is seen in Fig. 2 where the numerical solutions of (39) are shown for various initial radii. While all longitudinal case solutions merge toward the $u \sim r^{1 / 3}$ power-law there is a large spread in the transversal case solutions.

The longitudinal and transversal cases are the two limits for a general case where both kinds of scaling of the decay time scale occur. One can model the mixing of both cases by writing the dissipation time scale as

$\tau=k\left(\frac{r}{r_{0}}\right)^{\alpha}\left(\frac{u}{u_{0}}\right)^{2-\alpha}$

where $0<\alpha<1$ is a dimensionless parameter which determines the mixing. $\alpha=0$ corresponds to the pure longitudinal case and $\alpha=1$ to the transversal case.

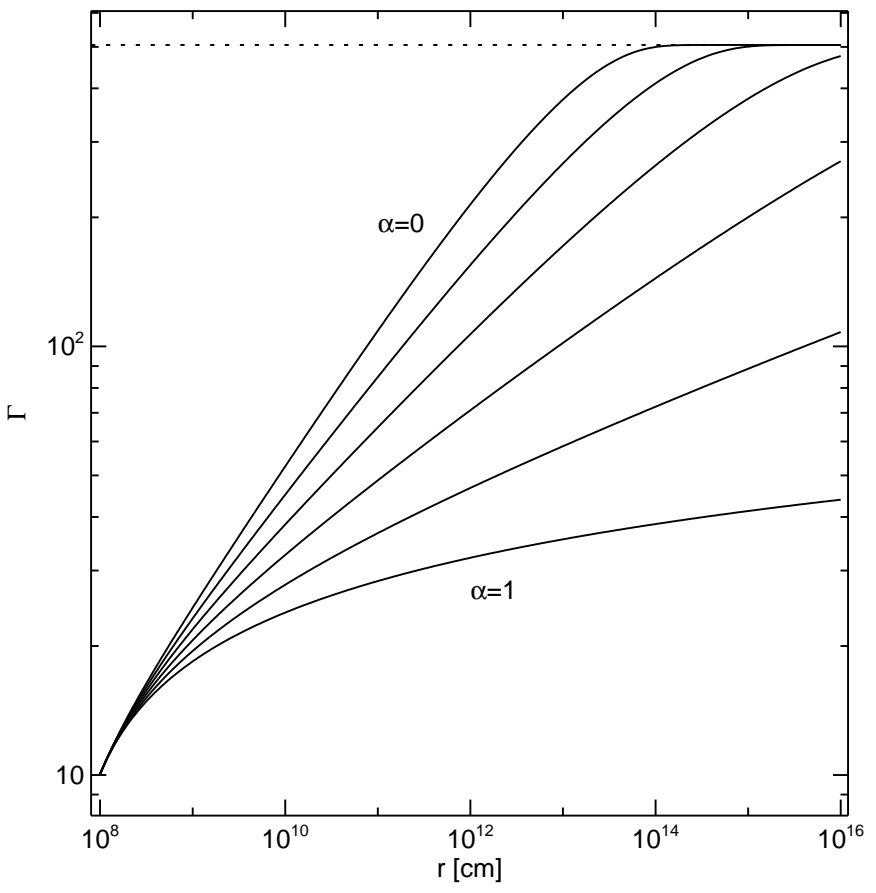

Fig. 3. The Lorentz factor $\Gamma$ for different parameters $\alpha \in$ $\{0,0.2,0.4,0.6,0.8,1\}$. The other wind parameters were set to $\sigma_{0}=100, \mu^{2}=0.5, \epsilon=0.1, r_{0}=10^{8} \mathrm{~cm}, \Omega=10^{4} \mathrm{~s}^{-1}$ (corresponding to $\vartheta=10.8^{\circ}$ in the transversal description). The different winds all start with the same dissipation rate so that they show the same initial acceleration. $\Gamma_{\infty}$ is marked by a horizontally dotted line.

The constant $k$ can be written depending on the corresponding model parameters as

$k=\frac{2 \pi \sigma_{0}}{\epsilon \Omega}$

or as

$k=\frac{\vartheta r_{0} \sqrt{\sigma_{0}}}{\epsilon c}$.

Figure 3 shows the velocity profiles for various $\alpha$ values. All graphs result from a numerical integration of (39). Beyond the photosphere, assuming it is around $10^{11} \mathrm{~cm}$, the dissipation is only efficient if the field variation does not point in transversal direction. The efficiency estimation in (59) is therefore an upper limit for the general case with $\alpha \neq 0$.

\subsection{The validity of the MHD condition}

Because we work with the ideal MHD approximation we have to make sure that there are enough charges in the flow to make up the required electric current density. Because the reconnection processes will destroy the ordered initial field configuration quickly it does not make much sense to consider this configuration throughout the flow. But one can at least estimate needed currents by looking at a sinusoidal wave in the equatorial plane. In Paper I we derived the limiting radius where the MHD condition breaks down by using a constant flow speed and 
assumed $\mu=0$. The condition that enough charges are available to carry the current is

$$
\frac{\Omega B}{4 \pi \Gamma^{2}}=\frac{2 \rho e c}{m_{\mathrm{p}}} .
$$

This yields the radius up to which the MHD approximation holds:

$$
\begin{aligned}
r_{\mathrm{MHD}} & =\frac{4 e \sqrt{\pi L}}{m_{\mathrm{p}} c^{3 / 2} \Omega} \frac{u / \sigma_{0}^{3 / 2}}{\sqrt{1-u / \sigma_{0}^{3 / 2}}} \\
& >4 \times 10^{18} \mathrm{~cm} \cdot L_{50}^{1 / 2} \Omega_{4}^{-1} \sigma_{0,2}^{-1}
\end{aligned}
$$

Here, we have used the dependence (21) of the magnetic field strength on velocity. In (66) $r_{\mathrm{MHD}}$ is written as a function of $u$ and depends implicitly on $r$. At $r_{0}$ where $u=u_{0}=\sqrt{\sigma_{0}}$ it starts at the value (67) and rises strongly until the final velocity $u=u_{\infty}=\sigma_{0}^{3 / 2}$ is reached where $r_{\text {MHD }}$ diverge to $\infty$. For the GRB parameters assumed here, we find $r_{\mathrm{MHD}} \gg r_{\mathrm{sr}}$ and the MHD approximation is always fulfilled, as in Paper I.

\subsection{Comparison with the striped pulsar wind}

Dissipation of magnetic energy was applied to the Crab pulsar wind by Lyubarsky \& Kirk (2001). Their model setup included a striped pulsar wind (Coroniti 1990) that is the equatorial wind of on inclined rotator. This is quite similar to our longitudinal case where all Poynting flux can decay so that $\mu=0$. The wind starts with $\Gamma=\sqrt{\sigma_{0}}$ and reaches $\Gamma_{\infty}=\sigma_{0}^{3 / 2}$ as in our model. Due to a difference approach to model the reconnection rate they obtain a flow acceleration of $\Gamma \sim r^{1 / 2}$ (Eq. (30) Lyubarsky \& Kirk 2001) which is faster than $\Gamma \approx u \sim r^{1 / 3}$ form (50).

The findings of Lyubarsky \& Kirk (2001) that the reconnection is inefficient for the Crab wind seems to contradict our result, that it efficiently accelerates the GRB outflow. The reason for that is the different initial Poynting flux values used for the Crab pulsar and in our study. $\sigma_{0}$ is the critical parameter controlling the final Lorentz factor and the spatial size of the accelerating wind.

Discussing the Crab pulsar wind in detail and speculating why reconnection fails is beyond the scope of the present paper. Instead, we simply take the flow parameter values $\sigma_{0,2}=400, \Omega_{4}=0.02$ from Lyubarsky \& Kirk (2001) and show that our model gives basically the same result as the striped wind model. However, see Yubarsky \& Eichler (2001) for a critical revision of the Crab pulsar wind parameters. Equation (50) yields for the 4-velocity at the observed termination shock at $r=3 \times 10^{17} \mathrm{~cm}$

$$
\frac{u}{u_{\infty}}=0.023 \epsilon_{-1}^{-1 / 3}, \quad \frac{u}{u_{0}}=920 \epsilon_{-1}^{-1 / 3}
$$

When the wind reaches its termination shock only a small fraction of the Poynting flux was converted. Though, due to the small amount of mass in the flow large acceleration occurs and the Lorentz factor increases by almost 4 orders of magnitude. This is the same result as obtained by Lyubarsky \& Kirk (2001). The different acceleration laws of the two models does not change the picture. The observed pulsar wind bubble is to small to allow for efficient reconnection.

The radius $r_{\mathrm{sr}}$ from (49) denotes the radius where the Poynting flux conversion ends. Its value scales with the third power of $\sigma_{0}$. Plausible Lorentz factors for GRB winds of around $10^{2}-10^{4}$ imply $\sigma_{0,2} \approx 0.2-5$ (or larger for $\mu>0$ ). This lowers $r_{\mathrm{sr}}$ by 6 orders of magnitudes compared to the Crab wind. Thus $r_{\mathrm{sr}} \lesssim 3 \times 10^{15} \mathrm{~cm}$ which is smaller than the radius $r_{\mathrm{a}} \approx 10^{16} \mathrm{~cm}$ where the flow runs into the ambient medium (Piran 1999). The requirement on $\sigma_{0}$ for the dissipation to take place inside a radius $r_{\mathrm{a}}$ can be expressed by (49) which yields an upper limit for the initial Poynting flux ratio:

$\sigma_{0} \lesssim 1100 \cdot\left(r_{\mathrm{a}, 16} \epsilon_{-1} \Omega_{4}\right)^{1 / 3}\left(\frac{1-\mu^{2}}{0.5}\right)^{-2 / 3}$.

For GRBs there is no size problem as for the Crab wind and Poynting flux can be efficiently converted.

\section{Discussion}

We have investigated the effect of dissipation of magnetic energy in a GRB outflow on the acceleration of the flow. Such dissipation is expected if the flow contains small scale changes of direction of the field for example when the flow is produced by the the rotation of a non-axisymmetric magnetic field. The dissipation is governed by the speed of fast reconnection, parameterised in our calculations as a fraction $\epsilon \approx 0.1$ of the local Alfvén speed in the flow.

Two possibilities for the field geometry in the outflow have been considered: a geometry where the changes in the small scale field direction occur along the bulk flow direction, and a geometry where the field variation is transversal to the flow direction. The first mentioned, longitudinal case is expected in the equatorial plane of an inclined rotator as in the "striped" pulsar wind model of Coroniti (1990). The second, transversal case can be associated with a polar outflow where the field line structure resembles a spiral. In both cases there are MHD instabilities (tearing and kink instabilities) which lead to reconnection processes. They differ only by the functional form of the reconnection time scale.

We find that in any case the process leads to a strong increase of the bulk Lorentz factor of the flow. This acceleration is due to the outward decrease of the magnetic pressure resulting from the field decay. At the same time, the dissipated energy can be released to large extend in the optically thin part of the flow beyond its photosphere, and can power most if not all of the prompt emission. This provides an alternative to the internal shock model.

The calculation is done for a stationary wind. Why this approximation is valid for highly variable objects like GRBs is not obvious. The duration of GRBs $t$ is of the order of a few seconds. One can approximate the wind as stationary within a source distance $c t \approx 10^{11} \mathrm{~cm}$. Thus the 
flow up to the photospheric radius is well described by a stationary description. Further out the time dependence of a real flow will become more important but that topic is beyond the scope of this work.

The outflow with transversal field variation contains some additional complications which does not occur in the longitudinal case. The dissipation time scale is proportional to the source distance. This results in a rapid energy dissipation near to the source and the velocity profile depends critically on the radius where the dissipation sets in. But this initial radius is hard to estimate from first principles.

We have used the spiral-like field geometry of a polar flow as pictured in Paper I to justify the existence of transversal field variations. This field geometry occurs for a polar outflow of an axisymmetric rotator. The following arguments give reasons why this field geometry is rather special and may not be important in a general. The kink instability leads to a break-down of the ordered spiral field configuration. After some Alfvén crossing times the field geometry will have changed so that the "longitudinal" dissipation time will become important while the "transversal" time scale grows large and can be neglected. On the other hand the rotator may not be perfectly aligned and non-axisymmetric field components are also present in the polar outflow. So, we probably have always longitudinal field variations in the flow so that the findings found in our treatment of the "longitudinal case" might be much more applicable and general.

We assume that the thermal energy flux is negligible compared to the kinetic and Poynting energy flux. The temperature is set to zero which simplifies the treatment and allows an analytical integration of the dynamic equations. Setting the thermal pressure gradient artificially to zero might appear to underestimate the acceleration. On the other hand the energy equation takes care that all released magnetic energy shows up in kinetic form. In fact, we overestimate the acceleration by doing so because the energy part converted into heat reduces the the gain of kinetic energy in the flow. Another physical argument explains why the flow stays cold: The acceleration expressed by the scaling of the Lorentz factor gives $\Gamma \sim r^{1 / 3}$ for our model. The release of magnetic energy must therefore also scale with $r^{1 / 3}$. In contrast to that, purely thermal acceleration by adiabatic cooling leads to more rapid flow acceleration where the Lorentz factor scales like $\Gamma \propto r$ (Paczyński 1986). Thus, heating proceeds slower than adiabatic cooling so that the thermal pressure gradient is not important compared to the magnetic pressure gradient which drives the flow. The reason why Lyubarsky \& Kirk (2001) find a faster acceleration of $\Gamma \sim r^{1 / 2}$ in a similar model lies in the different reconnection prescription and is not due to their inclusion of thermal pressure.

In the optically thin regime part of the dissipated energy radiates away. There, the model over-estimates the gain of kinetic energy. We cannot give arguments how much dissipated energy escapes as prompt radiation so that the total amount of released energy gives only an upper limit on the Lorentz factor.

The photospheric radius determines the lower limit on radius for the region in which non-thermal radiation is expected to originate. For typical GRB parameters describing the total luminosity, the baryon loading, the fraction of dissipatable energy and the reconnection rate one finds that a considerable amount of dissipation takes place in the optically thin region. Part of the dissipated energy is converted into non-thermal radiation. The remainder still leads to an acceleration of the flow. This acceleration is caused by the magnetic pressure gradient induced by the field dissipation. Since the acceleration continues outside the photosphere up to the radius where all the free magnetic energy is used up this non-thermal radiation is emitted from matter with different Lorentz factors. The observable spectrum in thus smeared out compared to a spectrum from a uniformly moving medium. For a more sound analysis of this topic one needs a model for the radiation process.

The Poynting flux conversion happens at radii $r \lesssim$ $10^{15} \mathrm{~cm}$ which is inside the distance $\approx 10^{16} \mathrm{~cm}$ where the GRB outflow is expected to run into the external medium. Thus, the Poynting flux can be converted efficiently. But by applying the model to the Crab pulsar wind we come to the same conclusions as Lyubarsky \& Kirk (2001): the conversion is inefficient since the observed pulsar wind bubble is to small to contain the whole region where reconnection takes place. For the Crab pulsar the assumed initial Poynting flux ratio is larger than for GRBs leading to a much longer reconnection phase. The presented model does not settle this Crab wind problem.

The most important parameter which controls the amount of energy dissipated beyond the photosphere is the initial Poynting flux to kinetic energy flux ratio. If its value is around 100 or greater much non-thermal, prompt emission is produced. If its value is of the order of 10 , however, all the Poynting flux energy is converted into kinetic energy and thermal radiation. Only prompt thermal emission and afterglow emission is expected in this case. The initial Poynting flux ratio is a measure for the baryon loading in a sense that a high baryon loading corresponds to a low initial Poynting flux ratio. Observations indicate that X-ray flashes and X-ray rich GRBs are very similar phenomena which probably differ only by the amount of baryon loading (Heise et al. 2001). In the context of our model, X-ray flashes can be associated with low initial Poynting flux ratios. In this case, the X-ray emission is thermal radiation from the photosphere. Increasing the initial Poynting flux ratio leads to the emission of nonthermal $\gamma$-rays in the optically thin region, thus producing X-ray rich and regular GRBs. If afterglows of X-ray flashes could be observed they would yield information about the connection to regular GRBs. Afterglows depend less strongly on the initial Poynting flux ratio but rather on the total luminosity of the outflow. Thus, X-ray flash afterglows should be similar to afterglows of regular GRBs 
according to our model. In a future work we will investigate the thermal emission more quantitatively.

The model predicts black-body radiation originating from the photosphere of the flow. We can calculate the radius of the photosphere and the Lorentz factor of the flow there. Together with the temperature one is able to calculate the luminosity if the thermal radiation. Since our approximation treats the flow as cold we cannot give quantitative results in this respect. Though, one finds that the Lorentz factor at the photosphere depends only weakly on the model parameters. Therefore, the observable temperature $k T_{\text {obs }}=\Gamma_{\mathrm{ph}} k T /(1+z)$ of the thermal component of a GRB depends primarily on the redshift $z$ and the temperature in the comoving frame $T$. This result simplifies the task to disentangle the effects of different model parameters on the temperature. A detailed, quantitative analysis of the thermal radiation will be done in a following study.

Acknowledgements. I thank H. C. Spruit for enlightening discussions and the critical reading of the manuscript.

\section{References}

Bateman, G. 1980, MHD Instabilities, 2nd ed. (Cambridge (Mass.): MIT Press)

Begelman, M. C., \& Li, Z.-Y. 1994, ApJ, 426, 269

Bekenstein, J. D., \& Oron, E. 1978, Phys. Rev. D, 18, 1809

Belcher, J. W., \& MacGregor, K. B. 1976, ApJ, 210, 498

Berezinskii, V. S., \& Prilutskii, O. F. 1987, A\&A, 175, 309

Beskin, V. S. 1997, Physics Uspekhi, 40(7), 659

Coroniti, F. V. 1990, ApJ, 349, 538
Daigne, F., \& Drenkhahn, G. 2002, A\&A, 381, 1066

Fenimore, E. E., Epstein, R. I., \& Ho, C. 1993, A\&AS, 97, 59

Goodman, J., Dar, A., \& Nussinov, S. 1987, ApJ, 314, L7

Heise, J., in 't Zand, J. J. M., Kippen, R. M., \& Woods, P. M. 2001, in Gamma-Ray Bursts in the Afterglow Era, Rome Workshop (Oct. 2000) [astro-ph/0111246]

Kluźniak, W., \& Ruderman, M. 1998, ApJ, 505, L113

Königl, A., \& Granot, J. 2002, ApJ, 574, in press [astro-ph/0112087]

Lithwick, Y., \& Sari, R. 2001, ApJ, 555, 540

Lyubarsky, Y., \& Eichler, D. 2001, ApJ, 562, 494

Lyubarsky, Y., \& Kirk, J. G. 2001, ApJ, 547, 437

Lyutikov, M. 2001, Phys. Fluids, 14, 963

Mészáros, P., \& Rees, M. J. 1997, ApJ, 482, L29

Michel, F. C. 1969, ApJ, 158, 727

Paczyński, B. 1986, ApJ, 308, L43

Petschek, E. N. 1964, in Proc. of an AAS-NASA Symp. on the Physics of Solar Flares, ed. W. N. Hess (Washington, DC: NASA), 425

Piran, T. 1999, Phys. Rep., 314, 575

Preece, R. D. 2000, AAS/High Energy Astrophysics Division, 32,3005

Ruffert, M., Janka, H.-T., Takahashi, K., \& Schaefer, G. 1997, A\&A, 319, 122

Spruit, H. C. 1999, A\&A, 341, L1

Spruit, H. C., Daigne, F., \& Drenkhahn, G. 2001, A\&A, 369, 694

Takahashi, M., \& Shibata, S. 1998, PASJ, 50, 271

Thompson, C. 1994, MNRAS, 270, 480

Usov, V. V. 1992, Nature, 357, 472

Weber, E. J., \& Davis, Jr., L. 1967, ApJ, 148, 217

Woods, E., \& Loeb, A. 1995, ApJ, 453, 583 